\begin{tabular}{lllllllllllllllllllllllllllllllll}
\hline$R$ & $E$ & $V$ & I & S & T & A & D & E & E & S & T & U & D & I & O & S & I & N & T & E & $R$ & N & A & C & I & O & N & A & L & $E$ & S
\end{tabular}

\title{
China y la seguridad energética
}

Martín Pérez Le-Fort

Este artículo está orientado a establecer cómo están vinculadas las necesidades energéticas chinas, su seguridad energética y la seguridad energética regional, y la situación de seguridad en el Este de Asia en un sentido amplio, estableciendo cómo este vínculo moldea la estrategia china en el área de la energía. En este análisis se establece que el incremento de la competencia estratégica entre Estados Unidos y China podría provocar que la política de seguridad energética de China profundice visiones con mayor acento en la geoestrategia y la seguridad nacional. En este sentido, el fortalecimiento de una política exterior multilateralista en China y su involucramiento en la cooperación energética regional generan expectativas positivas respecto de la evolución de dichas políticas, aunque esta tendencia es aún germinal. Esta dualidad solo podría cambiar a partir de una relación sino-norteamericana más constructiva en relación con el ámbito regional.

\section{INTRODUCCIÓN}

1

ara el 2010, Asia será el mayor consumidor de energía primaria del mundo. Asia ya importa el $60 \%$ de su petróleo desde el Medio Oriente y esa dependencia tiende a incrementarse. Los países asiáticos han visto este problema en términos estratégicos por largo tiempo y esto podría aumentar la posibilidad de conflictos en las disputas que han de producirse en la región si se mantiene esta visión geoestratégica ${ }^{1}$. Entre estos países, la República Popular China gene- ra una serie de interrogantes, tanto en el impacto del desarrollo económico sobre sus necesidades energéticas, como en la actitud que tomará dicho país en los conflictos limítrofes que involucren recursos energéticos.

Durante la década de los años noventa, China transitó desde la autosuficiencia energética hasta llegar a ser importador neto de petróleo desde 1993, fundamentalmente como resultado de su acelerado proceso de desarrollo económico

${ }^{1}$ Manning, Robert A., "The Asian Energy Predicament”, Survival, Vol. 42, № 3, otoño (boreal) 2000, p. 73. 
producto del proceso de reforma económica iniciado a fines de los años setenta. Este proceso, además de generar mayor interés por aumentar la producción nacional, acentuó la necesidad de garantizar un entorno de seguridad regional estable que garantizara su libre flujo. El sostenido incremento de la demanda de energía se ha manifestado en la forma en que China ha enfrentado su seguridad energética, es decir, cómo garantizar la adecuada disponibilidad de energía, en variadas formas, cantidades suficientes y a precios razonables ${ }^{2}$.

\section{El acelerado proceso de desarrollo económico acentuó la necesidad de garantizar la estabilidad de la seguridad regional.}

La necesidad de abastecerse regularmente desde los países productores, de incrementar la exploración costa afuera, de abrirse a la exploración e inversión extranjeras en este campo, de mejorar los vínculos con los países productores e invertir en ellos, entre otros aspectos, se ha llevado a cabo en forma sistemática por el gobierno chino. Sin embargo, el proceso no está exento de dificultades, ya que la dinámica de los equilibrios estratégi- cos regionales, principalmente en función de la competencia estratégica sino-norteamericana, el problema de Taiwán, la disputa por las islas Senkaku con Japón, la disputa por las islas Spratly con varios países del sudeste asiático y factores secundarios, como la amenaza del terrorismo en la provincia china de Xinjiang ${ }^{3}$, entre otros aspectos, enfrentan a China con algunos obstáculos que podrían poner a prueba su capacidad de garantizar su propio suministro energético en una eventual crisis.

China posee una base de producción energética interna que depende fuertemente del carbón que alcanza un 55\% de la producción total, y en menor medida, del petróleo con un 20\%; el gas natural con un $10 \%$; la energía proveniente de recursos hídricos con un $10 \%$; y la energía nuclear con un 5\%. Como hemos dicho, la necesidad de energía de China aumenta gradualmente y se prevé que el año 2020 importará el $60 \%$ del petróleo que necesita y el $30 \%$ del gas natural ${ }^{4}$. Este cambio de contexto ha hecho que el país redefina su perspectiva estratégica en un sentido amplio, tanto en su política energética en función de garantizar el suministro de recursos para su desarrollo, como en su visión estratégica respecto de los países productores de petróleo, las vías

\footnotetext{
${ }^{2}$ Khatib, Hisham, "Energy Security”, IV, en Goldemberg, José (ed.), World Energy Assessment: Energy and the Challenge of Sustainability, Programa de las Naciones Unidas para el Desarrollo (PNUD), Departamento of Economía y de asuntos sociales de las Naciones Unidas (UNDESA) y World Energy Council (WEC), p. 112.

${ }^{3}$ Burles, Mark, "Chinese Policy Toward Russia and the Central Asian Republics", edición electrónica, RAND Corporation, 1999, pp. 5-11

${ }^{4}$ Andrews-Speed, Philip, Xuanli Liao y Dannreuther, Roland, "The Strategic Implications of China's energy Needs", Adelphi Paper 346, International Institute for Strategic Studies, p. 6.
} 
por las cuales se transportan esos recursos, y las regiones sujetas a disputas limítrofes que poseen recursos energéticos, entre otros aspectos.

\begin{tabular}{|c|c|}
\hline TIPO DE RECURSO & $\mathbf{\%}$ \\
\hline Carbón & 55 \\
\hline Petróleo & 20 \\
\hline Gas Natural & 10 \\
\hline Hidropoder & 10 \\
\hline Nuclear & 5 \\
\hline
\end{tabular}

Estimación porcentual respecto del total de recursos energéticos para el año 2020. Fuente: Ni Weidou, Li Zheng, Xue Yuan, National Energy Futures Análysis and Energy Security Perspectives in China, Tsinghua University, Beijing, 2000, p. 4.

Las proyecciones de la demanda energética varían, pero en general se habla de un incremento de alrededor de $200 \%$ entre el 2000 y $2020^{5}$. Cabe señalar que a comienzos del período de reforma, China trató de compensar el aumento de la demanda energética mediante el incremento de la producción local de carbón, ya que China ocupa el tercer lugar en el tamaño de sus reservas y es el primer productor mundial de este recurso ${ }^{6}$. Sin embargo, los avances en este sentido no lograron compensar la tendencia deficitaria, que se hizo efectiva a principios de los años noventa.

\section{PRODUCCIÓN INTERNA DE CARBÓN}

China es el mayor productor y consumidor de carbón del mundo. En 1989 llegó a ser el principal productor y ha seguido con un fuerte crecimiento, aunque irregular ${ }^{7}$, hasta ahora en que alcanza cifras cercanas al $30 \%$ de la producción mundial. Cabe señalar, que algunas cifras chinas pueden resultar confusas ya que se refieren a carbón crudo, sucio. Por otra parte, hay algunas dificultades tecnológicas que permiten una tasa de recuperación de material relativamente baja en gran parte del sector ${ }^{8}$. El carbón ocupa cerca del $90 \%$ de las reservas de energía convencional de dicho país. En 1998, había alrededor de 72 mil minas de carbón, divididas en términos de propiedad en estatales claves (593), estatales locales (1640) y minas de pueblos y aldeas (alrededor de 70 mil). Las dos primeras proveían 4,1 millones de los 6,6 millones de empleos en esa industria. Sin embargo, esta es una industria que enfrenta dificultades en términos de competitividad e impacto ambiental ${ }^{9}$. La producción del recurso aumentó de $620 \mathrm{Mt}$. (millones de toneladas) en 1980, a 1116,4 Mt. en 1992, y 1397 Mt. en $1996^{10}$. Un problema que China debe enfrentar es la sobreproducción de este recurso, lo que impulsó al

${ }^{5}$ Ibid., p. 22.

${ }^{6}$ Ibid., p. 21.

${ }^{7}$ Smil, Vaclav, “China’s Energy and Resource Uses”, The China Quarterly, № 156, diciembre 1998, p. 937.

${ }^{8}$ Ibid., p 938.

${ }^{9}$ Wang Qingyi, Coal Industry in China: Envolvements and Prospects, Universidad de Tsinghua, Beijing, China. Comissioned Papers, The Nautilus Institute, 2002, p. 1.

${ }^{10}$ Ibid., p. 2. 
gobierno chino a disminuirla aplicando políticas tales como la búsqueda de mercados para la exportación (llegando a ser en 2002 el segundo mayor exportador del mundo) y el cierre de minas (principalmente pequeñas $)^{11}$. Se espera que en los próximos años aumente la demanda de carbón con lo cual deberá enfrentar un escenario de mayor competitividad, con mayores exigencias ambientales y una reforma y reorganización, agrupando las minas en siete corporaciones para 2005, e incorporando, además, al sector inversión extranjera y mejoras tecnológicas ${ }^{12}$.

\begin{tabular}{|c|c|}
\hline AÑO & $\begin{array}{c}\text { PRODUCCIÓN DE } \\
\text { CARBÓN NATURAL } \\
\text { (BT: MILES DE MILLONES } \\
\text { DE TONELADAS) }\end{array}$ \\
\hline 1995 & 1,36 \\
\hline 2000 & 1,4 \\
\hline 2010 & 1,7 \\
\hline 2020 & $2,0-2,1$ \\
\hline 2050 & $2,8-3,0$ \\
\hline
\end{tabular}

Estimación de la producción de carbón natural. Fuente: Ni Weidou, Li Zheng, Xue Yuan, National Energy Futures Analysis and Energy Security Perspectives in China, Universidad Tsinghua, Beijing, 2000, p. 5.

\section{Producción INTERNA DE PETRÓLEO}

En 2002 China fue el tercer mayor consumidor del mundo de petróleo y sus derivados, tras los Estados Unidos y Japón, con una demanda total de 5,26 millones de barriles por día. Sin embargo, se cree que a partir del año 2005 la demanda china superará a la japonesa. En 1998, China reorganizó las empresas de gas y petróleo del Estado en dos instancias principales focalizadas regionalmente: la China National Petroleum Corporation (CNPC) en el norte y oeste del país y la China Petrochemical Corporation (Sinopec) en el sur, aunque seguro inclinándose a la exploración y producción de gas y petróleo para la CNPC y de las actividades de refinación para Sinopec. A estos organismos, hay que agregar la China National Offshore Oil Corporation (CNOOC) que se orienta a la exploración y producción en la costa y a la creación en 1997 de una nueva compañía llamada China National Star Petroleum. La supervisión de la reglamentación de este sector está, desde comienzos del año 2003, a cargo de la State Energy administration $(\mathrm{SEA})^{13}$.

China necesita modernizar su infraestructura de transporte y almacenamiento. Por otra parte, la mayoría de los recursos internos chinos están bien explorados y los campos petroleros más grandes están produciendo para 30 años o más, como lo indican los pozos de Daqing en la provincia de Heilongjiang (desde 1958) y Shengli en la provincia de Shandong (desde 1962$)^{14}$. La región de Xinjiang, donde

${ }^{11}$ China, Country Analysis Briefs, Energy Information Administration (originario estadístico del Departamento de los Estados Unidos), <http://www.eia.doe.gov/emu/cabs/china.html>.

${ }^{12}$ Wang Qingyi, op. cit., p. 11, y China, Country Analysis Briefs, Energy Information Administration (Organismo estadístico de Departamento de energía de los Estados Unidos), <http://www.eia.doe.gov/ emu/cabs/china.html>.

${ }^{13}$ China, Country Analysis Briefs, op. cit.

${ }^{14}$ Smil, Vaclav, p. 944. 
se encuentra la Cuenca del Tarim, sería la última frontera en la explotación continental de hidrocarburos con participación más activa de empresas extranjeras en su exploración ${ }^{15}$.

La exploración frente a la costa solo aporta alrededor del $10 \%$ de la producción total, aunque las disputas limítrofes en el Mar del Sur de China y en el Mar de China Oriental han generado grandes expectativas aún no confirmadas. En el primer caso, la exploración de las islas Spratly (Nansha) se inició en 1987 y en 1992 comenzó la exploración por la empresa norteamericana Creston Energy Corporation; otros actores regionales están desarrollando procesos similares en dichas islas ${ }^{16}$.

\begin{tabular}{|l|c|c|c|c|}
\hline \multicolumn{1}{|c|}{ AÑO } & 2005 & 2010 & 2015 & 2020 \\
\hline \multicolumn{6}{|c|}{ *Millones de Toneladas } \\
\begin{tabular}{|l|c|c|c|c|}
\hline Producción & $155-$ & $155-$ & $175-$ & $130-$ \\
de petróleo & 188 & 195 & 225 & 200 \\
\hline Demanda de & $238-$ & $210-$ & $314-$ & $330-$ \\
petróleo & 277 & 371 & 437 & 575 \\
\hline
\end{tabular}
\end{tabular}

Resumen de las estimaciones de producción y demanda de petróleo a partir de cuadros elaborados por AndrewsSpeed, Philip, Xuanli Liao and Dannreuther, Roland, "The Strategic Implications of China's energy Needs", Adelphi Paper, 346, International Institute for Strategic Studies, pp. 25 y 29.

Las fuentes de petróleo que se están explorando en el interior del territorio chino se encuentran en la cuenca de Tarim, localizada en el noroeste del país, en la región autónoma de Xinjiang, que tiene reservas estimadas de $147 \mathrm{bb}$. Inicialmente, su exploración se limita a empresas estatales chinas, pero se ha abierto a la participación de capital y tecnología extranjeros. Cabe señalar que esta región tiene como característica un terreno complejo, con dunas de arena y viento que dificultan las tareas logísticas y de transporte ${ }^{17}$. Las importaciones de petróleo de China provienen principalmente del Medio Oriente y el sudeste asiático y en menor medida de África y otros mercados.

\begin{tabular}{|l|r|}
\hline País & $\%$ \\
\hline Omán & 13,7 \\
\hline Yemen & 11,3 \\
\hline Indonesia & 10,8 \\
\hline Irán & 10,8 \\
\hline Angola & 7,9 \\
\hline Arabia Saudita & 6,8 \\
\hline Reino Unido & 6 \\
\hline Noruega & 5,5 \\
\hline Vietnam & 4,1 \\
\hline Angola & 3,7 \\
\hline Otros & 19,4 \\
\hline
\end{tabular}

Fuentes principales de petróleo crudo según Fan Weixin, "China's Oil Trade Hits Record Highs in 1999", China Oil, Gas and Petrochemicals, Vol 8, $\mathrm{N}^{\mathrm{o}} 3$, febrero de 2000, p. 13.

\section{LA PRODUCCIÓN INTERNA DE GAS}

En China, la exploración sistemática del gas natural sólo comenzó en los años noventa $^{18}$. Se prevé que la utilización de gas natural en el país aumente de un 3\% en la actualidad a $8-10 \%$ en 2015 . China posee recursos de gas en la cuenca del Erdos, Sichuan, las cuencas del Tarim,

${ }^{15}$ Ibid., p. 942.

${ }^{16}$ Yann-huei Song, "United States and Territorial Disputes in the Soth China Sea: A Sstudy of Ocean Law and Politics", Maryland Series in Contemporary Asian Studies, No 1, 2002 (168), pp. 19-21.

${ }^{17}$ Salameh, Mamdouh G., "China, Oil and Risk of Regional Conflict", Survival, Vol. 37, N 4, invierno (boreal) 1995-96, pp. 138-141.

${ }^{18}$ Andrews-Speed, Philip, Xuanli Liao and Dannreuther, Roland, op. cit., p. 27. 
Junggar y Qaidam, así como en el Mar del Sur de China. Recientemente se ha incorporado el yacimiento de gas Chunxiao, en la cuenca del Xihu, en la costa próxima a Shanghai. Existe, además, un proyecto de gasoducto que vinculará la cuenca del Tarim con Shanghai, incorporado en el X Plan quinquenal 2001-2005 ${ }^{19}$.

Como consecuencia del incremento de la demanda de gas, China se encuentra en un proceso de expansión de su infraestructura, ya que actualmente este recurso sólo representa el 3\% del consumo total de energía. Las mayores reservas de esta clase de energía se encuentran al norte y oeste de China, lo que implica la necesidad de construir nuevos gasoductos. Uno de los proyectos más importantes al respecto vincula la provincia de Xinjiang, recogiendo también recursos de la cuenca del Ordos (en la región autónoma de Mongolia Interior) hasta llegar a Shanghai. Se espera que este gasoducto de $4200 \mathrm{~km}^{20}$ esté finalizado el año $2005 \mathrm{y}$ podría ser la base de futuros vínculos con Asia central. Existe un gasoducto que vincula Ordos y Beijing desde 1997, pero se cree necesario construir un segundo ${ }^{21}$. Otro proyecto podría unir Irkusk en $\mathrm{Ru}$ sia con China.

\begin{tabular}{|l|c|c|c|c|}
\hline \multicolumn{1}{|c|}{ AÑo } & 2005 & 2010 & 2015 & 2020 \\
\hline \multicolumn{4}{|c|}{ *Miles de millones de metros cúbicos por año } \\
\hline $\begin{array}{l}\text { Producción } \\
\text { de gas* }\end{array}$ & $3-40$ & $\begin{array}{c}10- \\
75\end{array}$ & $\begin{array}{c}20- \\
100\end{array}$ & $\begin{array}{c}40- \\
150\end{array}$ \\
\hline $\begin{array}{l}\text { Demanda } \\
\text { de gas* }\end{array}$ & $52-$ & $62-$ & $92-$ & $103-$ \\
67 & 121 & 200 & 266 \\
\hline
\end{tabular}

Resumen de las estimaciones de producción y demanda de gas a partir de cuadros elaborados por Andrews-Speed, Philip, Xuanli Liao and Dannreuther, Roland, "The Strategic Implications of China's energy Needs", Adelphi Paper, 346, International Institute for Strategic Studies, pp. 26 y 31.

LA PRODUCCIÓN DE ENERGÍA ELÉCTRICA

\section{A PARTIR DE FUENTES HÍDRICAS Y NUCLEARES}

Los recursos hidroeléctricos explotables de China se elevan a 378,5 GW. En el país se han construido más de 60.000 pequeñas estaciones hidroeléctricas con un fuerte impacto en la economía local y rural. China ha priorizado el desarrollo de plantas hidroeléctricas y un ejemplo de ello es la planta de las "Tres Gargantas", que tendrá una capacidad de 18,4 GW.

Por lo que toca a disminuir el impacto ambiental de las plantas a base de carbón, China ha acelerado la construcción de centrales nucleares en las regiones central y costera. El primer reactor de agua presurizada se instaló en 1992 en Qinshan, con una capacidad de $300 \mathrm{MW}$. En una segunda fase se han instalado dos reactores más con una capacidad de $600 \mathrm{MW}$

\footnotetext{
${ }^{19}$ Myers, Amy y Lewis Steven W., "Beijing's Oil Diplomacy”, Survival, Vol. 44, No 1, primavera (boreal) 2002, pp. 121-122.

${ }^{20} \mathrm{Ni}$ Weidou, Li Zheng, Xue Yuan, National Energy Futures Analysis and Energy Security Perspectives in China, de Universidad Tsinghua, Beijing, 2000, Commisioned Paper, The Nautilus Institute, <www.nautilus.org>, p. 5.
} 
cada uno. La tercera fase de Qinshan incluye dos reactores de agua pesada con una capacidad de $700 \mathrm{MW}$ cada uno. En 1999 se instalaron dos reactores de 900 MW en Daya Bay y otros dos de 100 MW en Linglao; actualmente se construyen dos reactores de agua presurizada en Lianyungang con una capacidad de $2000 \mathrm{MW}$. Se estima que para el 2006 la capacidad nuclear permitirá producir $20.000 \mathrm{MW}^{22}$.

\section{LA RELACIÓN CON LOS PAÍSES EXPORTADORES}

La vinculación energética de China con el sudeste asiático se ha incrementado también en términos positivos. Muestra de ello es la intensificación de los esfuerzos de los países de la ASEAN por diversificar sus fuentes energéticas y garantizar la cooperación energética regional con China (además de Japón y Corea del Sur). En dicha cooperación, el tema de la seguridad energética se ha hecho cada vez más relevante ${ }^{23}$. Cabe recordar también que Indonesia es un proveedor importante de recursos energéticos para China.

China mantiene vínculos energéticos con varios países del Medio Oriente, entre los que se cuentan Omán, Yemen, Irán, Emiratos Árabes Unidos y Arabia Saudita.
Las importanciones desde esos países alcanzaron los siguientes niveles:

\begin{tabular}{|c|c|c|}
\hline PAÍS & $\begin{array}{l}\text { AÑO } \\
1995\end{array}$ & $\begin{array}{l}\text { AÑO } \\
1999\end{array}$ \\
\hline \multicolumn{3}{|c|}{ *Miles de barriles por día } \\
\hline Omán & 73 & 100,4 \\
\hline Yemen & 49,4 & 82,6 \\
\hline Irán & 18,6 & 79 \\
\hline Emiratos Árabes Unidos & 7,2 & S.D. \\
\hline Arabia Saudita & 6,6 & 49,8 \\
\hline Medio Oriente & 155,2 & 348,2 \\
\hline Asia-Pacífico & 36,8 & 134,8 \\
\hline África & 141,6 & 136,4 \\
\hline Otros & 8 & 112,8 \\
\hline Total & 341,8 & 732,2 \\
\hline
\end{tabular}

Cuadro resumido de Myers Jaffe, Amy y Lewis Steven W., "Beijing's Oil Diplomacy", Survival, Vol. 44, No 1, primavera (boreal) 2002, p. 121.

EXPLORACIÓN E INVERSIÓN PETROLERA

en el Medio Oriente, Asia central, África y AmÉRICA LATINA

China ha desarrollado una diplomacia energética muy activa en diversas regiones, tales como África (Sudán y Nigeria), Asia central, (Kazajstán) y América Latina (Venezuela y Perú), internacionalizando así la industria del petróleo con actividades de exploración y adquisición $^{24}$.

La adquisición por partes iguales del yacimiento petrolífero de North Swing, en Alberta, Canadá por parte de la CNPC en julio de 1993, dio comienzo a la pro-

${ }^{21}$ China, Country Analysis Briefs, op. cit.

${ }^{22}$ Zhang Aling, Shi, Lin, "Prospects for and Impacts of Diversifyng Fuel use away Coal", Universidad de Tsinghua, Beijing, China. Commisioned Paper, The Nautilus Institute, <www.nautilus.org>, p. 13.

${ }^{23}$ Agence France Presse, julio 4, 2003, "ASEAN to Work with China, Japan, South Korea for Energy Security".

${ }^{24}$ Myers Jaffe, Amy y Lewis Steven W., op. cit., p. 122. 
ducción de petróleo chino desde ultramar $^{25}$. En septiembre de 1995, la CNPC obtuvo los derechos de explotación del block 6 en la cuenca del Muglad, en Sudán, seguida en marzo de 1997 por los blocks 1,2 y 4 de la misma cuenca ${ }^{26}$. En el Medio Oriente, la inversión en el yacimiento petrolífero de al-Ahdab, en Irak, generó algunas expectativas, aunque la reciente invasión estadounidense de dicho país seguramente alterará este proceso. Los vínculos con Arabia Saudita se profundizaron a partir de un acuerdo firmado en 1999 (aunque no incluía exploración y desarrollo) ${ }^{27}$.

\begin{tabular}{|l|l|l|r|}
\hline \multicolumn{1}{|c|}{ PAís } & ProYECTO & AÑo & $\begin{array}{c}\text { INVERSIÓN } \\
\text { (MILLONES } \\
\text { DE } \\
\text { DóLARES) }\end{array}$ \\
\hline Sudán & Blocks 1,2 y 4 & 1996 & 187,37 \\
\hline & Block 6 & 1997 & 3,02 \\
\hline & Refinería & 1997 & 56,2 \\
\hline Kazajstán & Arkbinsk & 1997 & 91,33 \\
\hline & Uzen & 1997 & 64,08 \\
\hline Venezuela & & 1997 & 82,59 \\
\hline Perú & Blocks 6 y 7 & 1997 & 6,14 \\
\hline Irák & Al-Ahdab & 1997 & 64,83 \\
\hline Nigeria & & 1997 & 26,09 \\
\hline Canadá & JV & 1997 & 1,8 \\
\hline Total & & & 583,63 \\
\hline
\end{tabular}

Cuadro resumido de Myers Jaffe, Amy y Lewis Steven W., "Beijing's Oil Diplomacy", Survival, Vol. 44, No 1, primavera (boreal) 2002, p. 123.

En octubre de 1997, China adquirió el $60.3 \%$ de la Aktyube Oil Company de Kazajstán, incluyendo producción y derechos de explotación en sus yacimientos petrolíferos ${ }^{28}$. China está tratando de ampliar sus vínculos energéticos con Rusia y el Asia central, principalmente Kazajstán. En el caso de Rusia, se proyecta construir un oleoducto que una el este de Siberia y el oriente de China, con un flujo estimado de 400 mil barriles diarios (las reservas de Yurubcheno-Takhomskaya), además de que hay conversaciones respecto de vínculos con las reservas de gas de Irkutsk, Yakutia y el este de Siberia ${ }^{29}$.

Respecto de Asia meridional, los contactos entre Sinopec y el gobierno de Pakistán, en noviembre de 2003, han abierto las puertas para la cooperación de empresas chinas y pakistaníes en el ámbito del petróleo y el gas $^{30}$.

América Latina es exportador neto de recursos energéticos (principalmente petróleo y carbón). En 1999, la región producía $8,8 \%$ del total de la energía mundial y consumía $6,7 \%{ }^{31}$. Respecto de esta región, China ha hecho inversiones en Venezuela y Perú. El Convenio de asociación sino-venezolano para la producción de bitumen (y sus fluidos anexos) entre la empresa Bitúmenes Orinoco S.A. (BITOR), filial de Petróleos de Venezuela S.A. (PDVSA) y la empresa china National Oil and Gas Exploration and Development

${ }^{25}$ China National Petroleum Corporation (CNPC), <http://www.cnpc.com.cn/english/gsgk/ lsyds_cnpcls.htm>.

${ }^{26}$ Ibid.

${ }^{27}$ Myers Jaffe, Amy y Lewis, Steven W., op. cit., pp. 122-124.

${ }^{28}$ China National Petroleum Corporation (CNPC), op. cit.

${ }^{29}$ Myers Amy y Lewis Steven W., op. cit., pp. 125-126.

${ }^{30} \mathrm{SINOPEC},<\mathrm{http}: / / \mathrm{www}$. sinopec.com.cn/english/en-newsevent/en-news/2624.html>. 
Corporation (CNODC), filial de la empresa china National Petroleum Corporation (CNPC), es ejemplo de ello. Este convenio se celebró el 6 de diciembre de 2001 y tiene una vigencia de alrededor de 30 años $^{32}$.

\section{Después de Estados Unidos, China es el consumidor más grande del mundo de recursos energéticos.}

El acuerdo apunta a la explotación de los recursos de la Faja del Orinoco, en donde se encuentran cuantiosas reservas probadas de bitúmenes naturales. El proyecto tiene por objeto diseñar, construir y operar un módulo de producción y emulsificación de bitumen natural para la elaboración de "orimulsión" (una emulsificación de bitumen natural suspendido en agua en presencia de surfactantes -con tecnología y marca vinculada a filiales de PDVSA)-- La capacidad nominal aproximada es de 7.250.000 toneladas métricas. Este proyecto incorpora también el transporte, exportación y comercialización de la "orimulsión" producida, todo ello sobre la base de las condiciones que detallará el referido Convenio de asociación. Las áreas específicas serán Cerro Negro, Estados Monagas y Anzoátegui. La par- ticipación inicial de las partes es $30 \%$ de BITOR y $70 \%$ CNODC. El convenio previene el control venezolano $(75 \%$ del capital social con derecho a voto, entre otros aspectos) ${ }^{33}$. La idea es que para el año 2004 , la producción anual alcance 6,5 millones de toneladas. Previamente, en julio de 1997, CNPC había obtenido derechos de explotación de los yacimientos petrolíferos venezolanos de Intercampo y Caracoles $^{34}$.

Por otra parte, CNPC, en octubre de 1993, adquirió los derechos de explotación y servicios del block 7 en yacimiento petrolífero de Talara en Perú y en julio de 1995 se sumó el block $6^{35}$. En 2001 las operaciones en este país produjeron 210 toneladas de crudo y 34,27 millones de metros cúbicos de gas natural ${ }^{36}$.

\section{LA SEguridAd ENERGÉTICA DE CHINA}

Las necesidades de seguridad energética han incrementado su prioridad en las necesidades estratégicas de China. El rápido crecimiento económico de la República Popular China, producido durante el período de la reforma, ha hecho que el país haya pasado desde la autosuficiencia a ser importador neto de recursos energéticos, siendo actualmente el segundo

${ }^{31}<\mathrm{http}$ //www.worldenergy.org/wec-geis/publications/reports/emt/overview/overview.asp $>$.

${ }^{32}$ El referido Convenio de asociación terminará en la fecha correspondiente al trigésimo aniversario de la realización exitosa de la prueba de funcionamiento de la planta de emulsificación de bitumen natural, o a los treinta y tres (33) años contados a partir de la inscripción de la Asociación en el Registro Mercantil, según cuál de estos sucesos ocurra primero.

${ }^{33}$ Petrolatin, <http://www.petrolatin.com/ci/legislacion/9268140.html>.

${ }^{34}$ China National Petroleum Corporation (CNPC), op. cit.

${ }^{35}$ Ibid.

${ }^{36}$ Ibid. 
consumidor más grande del mundo después de Estados Unidos. Esto ha alterado el interés por ciertas áreas de la periferia de China, poseedoras de dichos recursos. Ejemplos de ello son el creciente interés por los potenciales recursos energéticos de las islas Nansha (Spratlys) o los recursos procedentes de Asia central o que pasan por dicho territorio.

\section{Se han relegado a segundo plano las medidas relacionadas con el mercado.}

La creciente dependencia externa altera la política de total independencia energética, establecida durante el período de Mao, y está definiendo una nueva relación con el exterior. La política energética de Mao fue elaborada luego del descubrimiento del yacimiento petrolífero de Daqing en 1959, y el retiro de los asesores soviéticos de esta zona en 1960, como parte de la crisis de las relaciones sino-soviéticas en la década de los años sesenta. El país llegó a ser autosuficiente a mediados de los años sesenta y comenzó a exportar pequeñas cantidades del recurso en los años setenta ${ }^{37}$.

La perspectiva china respecto de la seguridad energética apunta principalmente a maximizar la producción interna de gas y petróleo, invertir en la producción de ultramar y aumentar los vínculos con los países exportadores. En este sentido, se está dando un enfoque estratégico en un sentido amplio a la política de seguridad energética, pero relegando a segundo plano las medidas de mercado, tales como la liberalización de los mercados internos o la disminución de la demanda. Por otra parte, recién comienzan a implementarse medidas de respuesta de emergencia a eventuales crisis $^{38}$. La creciente importancia de los suministros energéticos que son transportados desde países del Medio Oriente (entre los que se cuentan Omán, Yemen e Irán) hacia China, ha aumentado la importancia de las líneas de comunicación marítima que cruzan los estrechos del sudeste asiático hacia el Mar del Sur de China, generando la percepción de que Estados Unidos es la principal amenaza a su seguridad energética $^{39}$, lo que podría hacerla priorizar los vínculos energéticos continentales

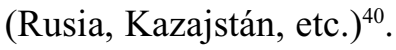

\section{LA SEGURIDAD ENERGÉTICA EN EL CONTEXTO ESTRATÉGICO REGIONAL}

Si vinculamos estas necesidades y opciones de seguridad energética con el contexto regional, encontramos una relación muy constructiva con un sudeste asiático incorporado a ASEAN, organismo que ha sido un impulsor de la integración energética regional y que ha adopta-

${ }^{37}$ Strecker Downs, Erica, "China's Quest for Energy Security”, publicación electrónica de RAND Corporation, 2000, p. 12.

${ }^{38}$ Andrews-Speed, Philip, Xuanli Liao and Dannreuther, Roland, op. cit., pp. 42-43.

${ }^{39}$ Strecker Downs, Erica, op. cit., p. 44.

${ }^{40}$ Ibid., pp. 45-46. 
do medidas a estos efectos desde la década de los años noventa. La opción regional de ASEAN de consensuar políticas orientadas a obtener seguridad, sustentabilidad y utilización eficiente de los recursos energéticos naturales se asemeja mucho a las políticas que en este ámbito realiza Japón, a lo que se suma un fuerte impulso a la construcción de redes energéticas trans-ASEAN. Debido al incremento de la demanda de combustibles fósiles, ASEAN busca aunar esfuerzos con esos países en la búsqueda de una mayor seguridad energética, articulada en torno al desarrollo del gas natural, las reservas de petróleo y la energía renovable ${ }^{41}$.

\section{Hay consenso en la importacnia de incorporar la sustentabilidad como eje de la seguridad energética.}

En el noreste asiático, el escenario es más heterogéneo. Rusia ha establecido una política de seguridad energética similar en muchos aspectos a la de China, es decir, orientada más hacia la interrupción física e impacto, que hacia aspectos de mercado, pero constituyéndose en un proveedor de recursos energéticos de primer orden y con una fuerte tendencia a incrementar los lazos energéticos regionales $^{42}$. La península coreana presenta ciertos contrastes entre Corea del Sur, que importa casi la totalidad de la energía que consume $^{43}$ y Corea del Norte, cuya producción energética se basa principalmente en una ineficiente utilización del carbón ${ }^{44}$. En este sentido, Rusia puede jugar algún rol en la situación de la península ya que existen negociaciones para el tránsito de gas desde Rusia, pasando por China e involucrando a ambas Coreas, lo que podría facilitar una mayor integración energética entre ambas. Por su parte, Japón carece de fuentes significativas de recursos energéticos y debe importar gran parte de estos recursos desde el exterior, aunque aplica una política muy equilibrada de seguridad energética que busca un adecuado equilibrio entre crecimiento económico, protección del medio ambiente y dicha seguridad energética ${ }^{45}$. Su sensibilidad a una eventual crisis en el Mar de Sur de China que afecte el transporte proveniente del Medio Oriente la obliga

${ }^{41}$ Presentación de Guillermo R. Balce, Christopher G. Zamora y Cecilya Malik en el Japan-ASEAN Energy Supply Security Planning Workshop 2001, Amari Hotel, Bangkok, Tailandia, 13 de septiembre de 2001 y en <http://www.aseanenergy.org/publications_statistics/apaec_1999-2004/apaec.htm>.

${ }^{42}$ Russia, Country Analysis Briefs, Energy Information Administration (Statistical Agency of the U.S. Department of Energy), <http://www.eia.doe.gov/emu/cabs/russia.html>.

${ }^{43}$ Chung, Woo Jin, "National Energy Futures Analysis and Energy Security Perspectives in the Republic of Korea”, Korea Energy Economics Institute, República de Corea, pp. 3-4, en South Korea, Country Analysis Briefs, Energy Information Administration (Statistical Agency of the U.S. Department of Energy), <http://www.eia.doe.gov/emeu/cabs/skorea.html $>$.

${ }^{44}$ North Korea, Country Analysis Briefs, Energy Information Administration (Statistical Agency of the U.S. Department of Energy), <http://www.eia.doe.gov/emeu/cabs/nkorea.html>.

${ }^{45}$ Hashimoto, Kohei, “Asia's Energy Security and the Role of Japan: A Diplomatic Perspective”, en Japanese Energy Security and Changing Global Energy Markets: An Analysis of Northeast Asian Energy 
a procurar activamente la estabilidad regional.

\section{Estados Unidos ha comenzado a distanciarse de su papel neutral}

\section{y ha aumentado su presencia militar en la región.}

En el contexto de APEC, se ha hecho patente la necesidad de abordar este tema de forma estratégica mediante el logro del consenso para aplicar medidas de corto plazo ante eventuales crisis y de largo plazo (pragmáticas y políticamente aceptables), destinadas a responder a las amplias amenazas que enfrenta el suministro de energía la región ${ }^{46}$. En APEC Hay claro consenso respecto de la importancia de incorporar la sustentabilidad como eje de la seguridad energética, reflejando la visión japonesa y de ASEAN, además de la estadounidense, aunque con evidente diferencia de acentos respecto de esta última. La amenaza del terrorismo ha involucrado a APEC en medidas de seguridad energética más tradicionales, aunque su preponderancia en el largo plazo no resulta tan clara.
En síntesis, a grandes rasgos hay dos tipos de políticas de seguridad energética. Una que apunta a visiones estratégicas asociadas a maximizar la seguridad nacional, en la que se encuentran China y Rusia, y otra que apunta a la necesaria vinculación entre desarrollo económico, sustentabilidad en un sentido amplio y seguridad energética, y que atribuye prioridad a la desregulación de los mercados y a la disminución del consumo. Las medidas de protección de la infraestructura siguen siendo un elemento común y el concepto que cobra mayor fuerza no solamente a nivel regional es la diversificación de las fuentes de recursos energéti$\cos$.

El escenario antes esbozado indicaba la existencia de un proceso de integración, pero marcado por dos visiones distintas de la seguridad energética. Si incorporamos el escenario estratégico regional, encontramos que la relación de competencia estratégica sino-norteamericana es un elemento determinante en la seguridad del este de Asia. Pese a que el tema del terrorismo ha generado expectativas de cooperación en la relación de ambos países, esto no ha alterado sustancialmente las opcio-

Cooperation and Japan's Evolving Leadership Role in the Region, May 2000. También en Suzuki, Tatsujiro, "Energy Security and the Role of Nuclear Power in Japan", Central Research Institute of the Electric Power Industry, pp. 15-16 y en The Ministry of Foreign Affairs of Japan, Strategy and Approaches of Japan's Energy Diplomacy, diciembre 2002, en Japan, Country Analysis Briefs, Energy Information Administration (Statistical Agency of the U.S. Department of Energy), <http://www.eia.doe.gov/emeu/ cabs/japan.html>, y en Suzuki, Tatsujiro, "Energy Security and the Role of Nuclear Power in Japan", Central Research Institute of the Electric Power Industry, pp. 15-16 y en The Ministry of Foreign Affairs of Japan, Strategy and Approaches of Japan's Energy Diplomacy, diciembre 2002.

46 "Energy Security Initiative" (México: Seminario de seguridad energética de APEC, 23 de julio de 2002), p. 1.

${ }^{47}$ East Asian Strategic Review, 2003, The National Institute for Defense Studies, Japán (Tokyo, The Japan Times, 2003), pp. 172-175. 
nes estrategicas de ninguno de ellos. Esto se debe en gran medida a la percepción china de la fuerte influencia estadounidense en la región ${ }^{47}$. Esta competencia se refleja en múltiples áreas, como por ejemplo, en el debate respecto del Sistema de Defensa de Misiles de Teatro (TMD), incluido en el sistema Nacional de Defensa de Misiles, $\mathrm{NMD}^{48}$. También se visualiza en los lazos de seguridad norteamericanos con Taiwán, con el agravante de que una eventual independencia de Taiwán y el posible respaldo militar de Estados Unidos, podría llevar a un conflicto abierto de este país con China. En este sentido, las disputas en el Mar del Sur de China, que involucran a China y varios países del sudeste asiático, son un espacio más sutil de competencia hegemónica sino-norteamericana. A largo plazo la opción estratégica china parece más orientada a reforzar su presencia regional, que a gatillar una crisis importante o bien crisis parciales en la zona, ya que podría tener consecuencias adversas para su flujo de importaciones energéticas y, por ende, para su propia seguridad energética.

Por su parte, el papel de Estados Unidos no está exento de ambigüedades. A la política respecto de Taiwán debemos sumar su interés en la solución pacífica de las disputas del Mar del Sur de China, ya que aunque no se involucra abiertamente en ellas, a partir del argumento de garantizar la libertad de navegación en la región, ha comenzado a distanciarse gradualmente desde un papel discursivamente neutral, incrementando además su presencia militar en el ámbito regional ${ }^{49}$. Las visiones optimistas, que ven el rol estadounidense como estabilizador de las líneas marítimas regionales, no aciertan a percibir la amenaza que esto podría representar a largo plazo para China.

\section{El conflicto con Taiwán podría afectar los esfuerzos de integración económica con Japón.}

Las crecientes necesidades energéticas de China han incrementado la importancia para el país de contextos regionales tales como el Mar del Sur de China, en el que ha desarrollado un mayor grado de asertividad, a fin de obtener y/o garantizar el flujo energético para su desarrollo económico. En un sentido amplio, el interés estratégico militar, dirigido hacia cómo abordar las inestabilidades regionales y disputas territoriales en la periferia sudeste y sudoeste ${ }^{50}$, ha otorgado a dicho Mar una importancia creciente, y tiene un vínculo importante con la seguridad energética de China.

Por su parte, el noreste asiático se presenta amenazante en función de un posible escalamiento de la crisis de la penín-

${ }^{48}$ Hook, Glenn D., Gilson, Julie, Hughes Cristopher W. y Dobson, Hugo, Japan's International Relations. Politics, Economics and Security, Londres, Routledge, 2001, p. 223.

${ }^{49}$ Tangredi, Sam J., Globalization and Maritime Power, Washigton D.C., National Defense University Press, 2002, p. 298.

${ }^{50}$ Nan, Li, “The PLA's Envolving Warfighting Doctrine, Strategy and Tactics, 1985-1995: A Chinese Perspective.", The China Quarterly, No 146, junio 1996, p. 443. 
sula coreana gatillada por una mezcla de opciones militaristas y nacionalistas, de necesidades de seguridad energética y de subsistencia política del régimen comunista de Corea del Norte. Los esfuerzos externos por lograr un desarrollo energético de dicho país, sin que ello esté asociado a un programa nuclear de uso militar, no parecen haber dado los resultados esperados, $\mathrm{y}$ han transformado un problema que inicialmente fue de seguridad energética en un mecanismo de chantaje para obtener ayuda exterior. China requiere estabilizar dicha zona y, dentro de los múltiples medios que posee para presionar a Corea del Norte, están sus vínculos energéticos con ella o los que pueda desarrollar en el futuro ${ }^{51}$.

\section{Para enfrentar su déficit energético debe mejorar la relación con los países productores.}

Por otra parte, la integración económica de China con Japón no parece presagiar un incremento de tensiones respecto de su disputa por las islas Senkaku y esta más parece orientarse en el largo pla- zo hacia una explotación conjunta de los recursos energéticos que estas posean. $\mathrm{La}$ evolución del conflicto con Taiwán no garantiza sin embargo que estos lazos no se vean afectados en alguna medida. Este conflicto puede afectar profundamente el contexto regional así como los esfuerzos de integración económica, además de neutralizar algunos intentos de explotación conjunta de recursos energéticos en el Estrecho de Formosa ${ }^{52}$.

A partir de lo anterior se ha producido una alteración de la perspectiva de la Republica Popular China respecto de los esquemas de seguridad multilateral regional. China está abandonando su política vaga y cautelosa respecto de los esquemas de seguridad multilateral, buscando un mayor grado de participación. Esto se debe fundamentalmente a que su objetivo de construir un mundo multipolar pasa por contar con estructuras multilaterales que contrapesen la fuerte presencia estadounidense en el este de Asia, lo que se comprueba en la activa participación de China de la Organización de Cooperación de Shanghai en el Foro Regional de la ASEAN $^{53}$.

${ }^{51}$ Pollack, Jonathan D., "China and a Changing North Korea: Issues, Uncertainties, and Implications”, trabajo prepado para la conferencia "North Korea's Engagement-Perspectives, Outlook, and Implications", coauspiciada por the Federal Research Division, Biblioteca del Congreso el National Intelligence Council, Washington, DC, febrero 2001. También en Kim, Samuel S., "The Making of China's Korea Policy in the Era of Reform", en Lampton, David M. (ed.), The Making of Chinese Foreign and Security Policy in the Era of Reform, 1978-2000, Stanford, Stanford University Press, 2001, pp. 371-408.

${ }^{52}$ Hook, Glenn D., Gilson, Julie, Hughes Cristopher W. and Dobson, Hugo, Japan's International Relations. Politics, Economics and Security, Londres, Routledge, 2001, p. 223, en Strategic Survey, The International Institute for Strategic Studies, Londres, Oxford University Press, 2003, pp. 263-266. También en Jaustin, Greg and Harris, Stuart, Japan and Greater China, Honolulu, University of Hawaii Press, 2001, pp. 100-102.

${ }^{53}$ Pérez Le-Fort, Martín, "El Foro Regional de la ASEAN y la República Popular China”, Estudios Internacionales, $\mathrm{N}^{\circ} 143$, octubre-diciembre 2003, pp. 103-126. También en Pérez Le-Fort, Martín, 


\section{Conclusiones}

Como hemos visto, a largo plazo China mantendrá una fuerte dependencia energética del carbón, aunque enfrentando dificultades en términos de la mayor competitividad que necesita para enfrentar su incorporación a la OMC. Además, deberá enfrentar mayores exigencias medioambientales y la necesidad de una reorganización del sector, agrupando las minas en torno a un grupo reducido de corporaciones. A ello habrá que sumar la necesidad de una mayor inversión extranjera y mejoras tecnológicas al sector que incorporen una producción más limpia.

Si se acepta que China pasará de ser el segundo consumidor de petróleo mundial en el año 2003 y que en 2020 más de la mitad de su petróleo y la tercera parte del gas serán importados, nos encontramos ante un escenario poco alentador y con una situación que requiere medidas urgentes. El gobierno chino ha adoptado una política de reorganización administrativa que incluye ese sector, pero focalizándola regionalmente. A ello se han sumado los esfuerzos de exploración y explotación costa afuera, con muchas expectativas aunque con un aporte de solo el $10 \%$ de la producción total. En este sentido, las esperanzas puestas en el Mar del Sur de China aún no han dado frutos. Por otra parte, la expansión de la producción local, principalmente desde la cuenca del Tarim, no lograría compensar el déficit. La opción por incrementar la producción de energía desde fuentes hídricas y nucleares continuará teniendo baja incidencia.

\section{La relación positiva con los países de la ASEAN favorece una estabilidad que contrapese la presencia estadounidense en el sudeste asiático.}

Una alternativa de acción obvia ante la imposibilidad de enfrentar el déficit con producción interna es mejorar la relación con los países productores y adoptar medidas similares a las políticas de seguridad energética de otros actores, tales como Estados Unidos o la Unión Europea, en el sentido de procurar la diversificación de los países que lo abastecen de recursos energéticos. Actualmente, estos provienen principalmente del Medio Oriente de algunos Estados que el gobierno de Estados Unidos considera "parias" y el sudeste asiático, y la tendencia se orienta hacia una ampliación de los vínculos energéticos con Rusia y Kazajstán. En esta opción parece también involucrada cierta opción geoestratégica, ya que podría implicar costos mayores, pero mayor seguridad. Una opción complementaria para China ha sido la adopción de un programa de inversión externa en el sector energético en países tan variados como Sudán, Kazajstán, Venezuela, Perú, Canadá, Irak y Nigeria.

La visión de seguridad energética china tiene un fuerte acento en la maximi- 
zación de la producción interna de combustibles fósiles, inversión en la producción de ultramar y aumento de los vínculos con los países exportadores. Por otra parte, es evidente que las medidas de desregulación de mercado parecen no ser aún alternativas políticamente aceptables, aunque resulta curioso que no se hayan adoptado medidas orientadas a disminuir el consumo. Las medidas para enfrentar crisis transitorias tales como la acumulación de reservas estratégicas y otras de respuesta de emergencia parecen ser la opción natural en la etapa siguiente.

A largo plazo, los esfuerzos por fortalecer los lazos energéticos continentales (Rusia y el Asia central) permitirían disminuir la dependencia de los recursos provenientes del Medio Oriente y la sensbilidad respecto de las amenazas de su transporte por vía marítima. Los recursos provenientes de Rusia o Kazajstán estarían menos amenazados por una eventual crisis con Estados Unidos. Por otra parte, es compatible con la diversificación de sus fuentes de recursos energéticos ante eventuales crisis de alguno de sus proveedores.

Por otra lado, una relación positiva con los países de ASEAN y sus instancias apunta a construir una estabilidad amplia que contrapese la reconstitución de una fuerte presencia estadounidense en el sudeste asiático, algo debilitada durante la post Guerra Fría. El Foro Regional de la ASEAN, por su flexibilidad, por el bajo grado de formalización y por la percepción heterogénea de los distintos actores respecto de China, se presenta como una instancia oportuna para garantizar una base de estabilidad de la situación estra- tégica regional, abriendo además espacios de negociación y de explotación conjunta de recursos, pero sin discutir directamente temas de soberanía (que se mantienen en el plano bilateral).

El incremento de la competencia estratégica entre Estados Unidos y China podría conducir a que la seguridad energética de esta se oriente hacia visiones que privilegian lo geoestratégico y de seguridad nacional. En este sentido el fortalecimiento de visiones multilateralistas, con grandes expectativas respecto de la integración económica regional tales como APEC o una mayor integración en las instancias de ASEAN, permitiría disminuir este riesgo. Sin embargo, la opción entre geoestrategia y geoeconomía desde el punto de vista chino, parecería estar muy lejos de ser resuelta y la preferencia hacia la primera parece primar en muchas de sus decisiones. Esto sólo podría cambiar a partir de una relación sino-norteamericana más constructiva.

\section{BiBLIOGRAFÍA}

1. Manning, Robert A., "The Asian Energy Predicament", Survival, Vol. 42, No 3, otoño (boreal) 2000.

2. Khatib, Hisham; "Energy Security", Chapter IV; en: Goldemberg, José (ed.), World Energy Assessment: Energy and the Challenge of Sustainability, Programa de las Naciones Unidas para el Desarrollo, Departamento de Asuntos Económicos y Sociales, y World Energy Council (WEC).

3. Burles, Mark, "Chinese Policy Toward Russia and the Central Asian Republics", edición electrónica, RAND Corporation, 1999.

4. Andrews-Speed, Philip, Xuanli Liao and Dannreuther, Roland, “The Strategic Implica- 
tions of China's Energy Needs", Adelphi Paper, 346, International Institute for Strategic Studies.

5. Smil, Vaclav, "China's Energy and Resource Uses", The China Quarterly, № 156, diciembre 1998.

6. Wang Qingyi, Coal Industry in China: Envolvements and Prospects, Universidad de Tsinghua, Beijing, China, Comissioned Papers, The Nautilus Institute, 2002.

7. China, Country Analysis Briefs, Energy Information Administration, Statistical Agency of the U.S. Department of Energy, <http:// www.eia.doe.gov/emu/cabs/china.html $>$.

8. Yann-huei Song, "United States and Territorial Disputes in the Soth China Sea: A Sstudy of Ocean Law and Politics", Maryland Series in Contemporary Asian Studies, No 1, 2002 (168).

9. Salameh, Mamdouh G., "China, Oil and Risk of Regional Conflict”, Survival, Vol. 37, N 4, invierno (boreal) 1995-96.

10. Myers, Amy y Lewis Steven W., "Beijing's Oil Diplomacy", Survival, Vol. 44, No 1, primavera (boreal) 2002.

11. Ni Weidou, Li Zheng, Xue Yuan, National Energy Futures Análysis and Energy Security Perspectives in China, Universidad de Tsinghua, Beijing, 2000, Commisioned Paper, The Nautilus Institute, <www.nautilus.org $>$.

12. Zhang Aling, Shi, Lin, "Prospects for and Impacts of Diversifyng Fuel use away Coal", Tsinghua University, Beijing, China. Commisioned Paper, The Nautilus Institute, $<$ www.nautilus.org $>$.

13. Agence France Presse, 4 de julio de 2003, "ASEAN to Work with China, Japan, South Korea for Energy Security".

14. China National Petroleum Corporation (CNPC), <http://www.cnpc.com.cn/english/ gsgk/lsyds_cnpcls.htm>.

15. SINOPEC, <http://www.sinopec.com.cn/ english/en-newsevent/en-news/2624.html>.

16. <http://www.worldenergy.org/wec-geis/ publications/reports/emt/overview/ overview.asp $>$.

17. Petrolatin, <http://www.petrolatin.com/ci/ legislacion/9268140.html>.

18. Strecker Downs, Erica, "China's Quest for Energy Security”, publicación electrónica de RAND Corporation, 2000.
19. Nye, Joseph; "Energy and Security", Chapter 1, en Desse, David y Nye, Joseph (eds.), Energy and Security, Cambridge, Massacusetts: Ballinger Publishing Company, 1981.

20. Russia, Country Analysis Briefs, Energy Information Administration (Statistical Agency of the U.S. Department of Energy), <http:// www.eia.doe.gov/emu/cabs/russia.html $>$.

21. Corea del Sur, Country Analysis Briefs, Energy Information Administration (Statistical Agency of the U.S. Department of Energy), <http:// www.eia.doe.gov/emeu/cabs/skorea.html>.

22. Chung, Woo Jin, National Energy Futures Analysis and Energy Security Perspectives in the Republic of Korea, Korea Energy Economics Institute, República de Corea.

23. Corea del Norte, Country Analysis Briefs, Energy Information Administration (Statistical Agency of the U.S. Department of Energy), $<$ http://www.eia.doe.gov/emeu/cabs/ nkorea.html>.

24. Japón, Country Analysis Briefs, Energy Information Administration (Statistical Agency of the U.S. Department of Energy), <http:// www.eia.doe.gov/emeu/cabs/japan.html $>$.

25. Hashimoto, Kohei, "Asia's Energy Security and the Role of Japan: A Diplomatic Perspective", en Japanese Energy Security and Changing Global Energy Markets: An Analysis of Northeast Asian Energy Cooperation and Japan's Evolving Leadership Role in the Region, mayo 2000.

26. Ministerio de Relaciones Exteriores de Japón, Strategy and Approaches of Japan's Energy Diplomacy, diciembre 2002.

27. Suzuki, Tatsujiro, "Energy Security and the Role of Nuclear Power in Japan", Central Research Institute of the Electric Power Industry.

28. Taiwán, Country Analysis Briefs, Energy Information Administration (Statistical Agency of the U.S. Department of Energy), <http:// www.eia.doe.gov/emu/cabs/taiwan.html $>$.

29. Exposición de Balce, Guillermo R., Christopher G. Zamora y Cecilya Malik, en el Seminario Japan-ASEAN Energy Supply Security Planning 2001, Amari Hotel, Bangkok, Tailandia, 13 septiembre de 2001. 
30. <http://www.aseanenergy.org/publications_statistics/apaec_1999-2004/apaec.htm>.

31. Agence France Presse, 4 de julio de 2003, "ASEAN to Work with China, Japan, South Korea for Energy Security".

32. "Energy Security Initiative", Seminario de seguridad energética de APEC, México, 23 de julio de 2002.

33. East Asian Strategic Review, 2003, The National Institute for Defense Studies, Japón, Tokio, The Japan Times, 2003.

34. Tangredi, Sam J., Globalization and Maritime Power, Washigton D.C., National Defense University Press, 2002.

35. Jin, Canrong, "Uncertainties Caused by Violation of Tradition", Contemporary International Relations, China Institute of Contemporary International Relations, Vol. 13, $\mathrm{N}^{\circ}$ 9, septiembre 2003.

36. You, Ji, “A Test Case for China's Defence and Foreign Policies", Contemporary Southeast Asia, Vol. 16, № 4, marzo 1995.

37. Godwin, Paul H. B., "From Continent to Periphery: PLA Doctrine, Strategy and Capabilities Towards 2000", The China Quarterly, № 146, junio 1996.

38. Burles, Mark and Shulsky, Abram N., "Patterns in China's Use of Force: Evidence from History and Doctrinal Writings", edición electrónica, RAND Corporation, 2000.

39. Nan, Li, "The PLA's Envolving Warfighting Doctrine, Strategy and Tactics, 1985-1995: A Chinese Perspective", The China Quarterly, $\mathrm{N}^{\mathrm{o}}$ 146, junio 1996.

40. Cole Bernard D., "China's Maritime Strategy", en: "People's Liberation Army after Next", Puska, Susan M. (ed.). Strategic Studies Institute, U.S. Army War College, 2000.

41. Shee Poon Kim, "The South China Sea in the China's Strategic Thinking", Contemporary Southeast Asia, Vol. 19, № 4, marzo 1998.

42. Yang, Bojinag, "The Change and Posture in the Security situation in Northeastern Asia", Contemporary International Relations, Vol. 13, $\mathrm{N}^{\circ} 5$, mayo 2003.

43. Diario del Pueblo, <http://english. peopledaily.com.cn/200308/18/eng20030818_122485.shtml>.
44. Hook, Glenn D., Gilson, Julie, Hughes Cristopher W. y Dobson, Hugo, Japan's International Relations. Politics, Economics and Security, Londres, Routledge, 2001.

45. Strategic Survey, The International Institute for Strategic Studies, Londres, Oxford University Press, 2003.

46. Jaustin, Greg, y Harris, Stuart, Japan and Greater China, Honolulu, University of Hawaii Press, 2001.

47. Pollack, Jonathan D., "China and a Changing North Korea: Issues, Uncertainties, and Implications", listado presentado en la Conference "North Korea's EngagementPerspectives, Outlook, and Implications", compilado por la Federal Research Division, Biblioteca del Congreso y el National Intelligence Council, Washington, DC, febrero 23, 2001.

48. Kim, Samuel S., "The Making of China's Korea Policy in the Era of Reform", en Lampton, David M. (dir.), The Making of Chinese Foreign and Security Policy in the Era of Reform, 19782000, Stanford, Stanford University Press, 2001.

49. Vice Foreign Minister Wang Yi, Head of the Chinese Delegation to the Six-Party Beijing Talks, Receives a Joint Interview by Xinhua News Agency, People's Daily, CCTV, CRI and China Daily, 2003/08/26, <http:/www.fmprc.gov.cn/eng/gjwt/gjrdwt/t25372.htm>.

50. Anderson, Jennifer, "The Limits of Sino-Russian Strategic Partnership”, Adelphi Paper, 315, 1998, International Institute for Strategic Studies.

51. Pérez Le-Fort, Martín, Chacón, Alejandra y Toro, Agustín, "El Grupo de los 5 de Shanghai", Revista Politica y Estrategia, $\mathrm{N}^{\circ} 86$, Academia Nacional de Estudios Políticos y Estrategicos (ANEPE), Santiago, enero-abril, 2002.

52.- Pérez Le-Fort, Martín, Chacón Alejandra y Toro, Agustín, "La República Popular China y el Conflicto con Taiwán: Un Estrecho Margen de Maniobra", Estudios Internacionales, Año XXXIV, enero-marzo 2001, $\mathrm{N}^{\circ} 133$.

53. Constitución de la República Popular China, Beijing, Ediciones en lenguas Extranjeras, 1983. 